\title{
Group maintenance policy for mechanical products based on age
}

\section{replacement}

\author{
Jia Xinjian ${ }^{1, a}$, Wang Gong ${ }^{1}$, Shi Jianming ${ }^{1, b^{*}}$, Zhang Mengying ${ }^{1}$ \\ 'Department of Safety, Reliability and Maintainability Assurance \\ Technology and Engineering Center for Space Utilization, Chinese Academy of Sciences \\ Beijing,China \\ ajiaxinjian14@csu.ac.cn \\ ${ }^{\mathrm{b}} \mathrm{sjm} @ \mathrm{csu} . \mathrm{ac} . \mathrm{cn}$
}

Key words: maintenance support; group maintenance policy; start-up cost

Abstract:Systems in engineering field are becoming more and more complex nowadays, so how to exert maintenance support for complex systems is a critical problem. As the failure rate of most mechanical products is based on age, the replacement before the failure occurs is an effective way, by which the failure cost can be avoided, and this policy is called age replacement policy. However, with the systems are much more complicated, age replacement policy for a single component cannot meet the demand for maintenance support of complex systems, so it is necessary to maintain several components simultaneously, and this policy is called group maintenance policyin this paper. Group maintenance policy is of great importance for reducing the start-up cost and improving the availability of the system. In this paper, the method of calculating the optimal time for replacement for a group of components and the condition of the group maintenance policyis the optimal choice are introduced. In last chapter, a simulation software named VISUALSPAR is used to validate the correctness of the method, and a numerical example is presented to explain under what condition the group maintenance policy is the optimal choice.

\section{Introduction}

The failure's features of mechanical productions are: with the using time increasing, the failure rate is increasing because of abrasive wear, so the failure rate is an increasing function about using time (IFR). In engineering field, it is a common fact that the cost caused by failure is much expensive than the cost caused by replacement. So, in order to reduce the cost of maintenance support, people always replace the components that are going to be failed. This policy can increase the mean time to failure (MTTF) of the component, which is called age replacement policy.

Study for age replacement policyhas a long history in literature. Cox D R. studied if there exists an optimal time point, the component has to meet some requirements[1]. Glasser G J. shows us the method of calculating the optimal time point under different distributions[2]. Age replacement with continuous discounting was proposed in [3]. More general models were provided successively in $[4,5]$.

However, the mentioned achievements above concern only on a single unit, while in the engineering field, a system can be very complex. It may contain hundreds of components that are subject to different distributions. If the optimal time point of the components are calculated 
respectively, then many optimal time points are got, which means the system must be shut down over and over again for maintenance. Many systems such as a generator may have a cost every time the system is started, which is called start-up cost[6]. When a system has a start-up cost, it is very expensive if the system is shut down many times. Under this condition, if several components are maintained simultaneously at a same time point, this problem can be solved. This time point may not be the optimal time point for each single component, but it is the optimal time for maintenance of the system, i.e. if several components must be maintained at the same time, the maintenance cost is minimal if they are replaced at this time, and this policy is called group maintenance policy. In recent years, group maintenance policy is becoming more and more popular because of its advantages[7].

In this paper, the meaning of age replacement policy and how to calculate an optimal time point for maintenance if it exists will be introduced in Section 2. The method of calculating a system's optimal maintenance time point is introduced in Section 3. In Section 4, a numerical example is presented to validate the correctness of age replacement policy by a simulation software, by the way, calculate when the group maintenance policy is better than age replacement policy for a system.

\section{Age Replacement Policy for a Single Component}

When a component's failure rate increases as time goes by, it is an important problem to determine when to replace the component before failure. This is called age replacement policy, which will be further introduced in following chapters.

First, defining some parameters below:

$\mathrm{C}_{1}$ the cost caused by failure of the component

$\mathrm{C}_{2}$ the cost caused by replacement of a not failed component $\quad \mathrm{C}_{2}<\mathrm{C}_{1}$

$\mathrm{EN}_{1}(\mathrm{t})$ failure times of the component during the life cycle

$\mathrm{EN}_{2}(\mathrm{t})$ replacement times of the component during the life cycle

Based on the definitions above, cost of life cycle $t$ can be expressed as Eq.1:

$\hat{C}(t)=C_{1} * E N_{1}(t)+C_{2} * E N_{2}(t)$.

On the basis of the work done by Richard R.Barlow, we can get the result of Eq.1 easily[8]. The cost rate per unit of time is:

$\mathrm{C}(\mathrm{T})=\frac{C_{1} * F(T)+C_{2} * \bar{F}(T)}{\int_{0}^{T} \bar{F}(T) d t}$.

Where $\mathrm{F}(\mathrm{t})$ is the cumulative distribution function of the component, $\bar{F}(T)=1-F(T)$

Differentiating $\mathrm{C}(\mathrm{T})$ in Eq. 2 with respect to $\mathrm{T}$ and putting it equal to zero.

$\lambda(\mathrm{T}) \int_{0}^{T} \bar{F}(T) d t-F(t)=\frac{C_{2}}{C_{1}-C_{2}}$. 
Where $\lambda(\mathrm{T})$ is the failure rate of the component, $\lambda(\mathrm{T})=\frac{f(T)}{\bar{F}(T)}, f(T)=\dot{F}(T)$

The time point $\mathrm{T}^{*}$ that satisfied Eq.3 is the optimal time point for a single component replacement. If replacementsare exerted at $\mathrm{k} \mathrm{T}^{*}$ after the component is installed or at failure, the cost of maintaining the component is minimal.

From the literature [9], conditions of whether an optimal time is existed can be known.Defining $\mathrm{K}=\frac{C_{1}}{u\left(C_{1}-C_{2}\right)}, \mathrm{u}$ is the expected value of $\mathrm{F}(\mathrm{t})$, when the failure rate of the component is increasing respect to the using time, there is a theorem:

\section{Theorem 1:}

i) If $\lambda(\infty)>K$, then there exist an optimal time point $T^{*}$ for maintenance that satisfied the Eq.3.

ii) If $\lambda(\infty) \leq K$, then $T^{*}=\infty$,i.e., a component is replaced only at failure.

Using theorem 1, age replacement policy for a single component can be easily got.

\section{Multi-Component Group Maintenance Policy}

The Method of Calculating the Replacement Time of a Multi-Component System.In Section 2, age replacement policy of a single component is introduced simply, then it is extended in the following sections.

Suppose that a system contains $\mathrm{n}$ different components, these components all have an increasing failure rate respectively. Group maintenance policy is applied to find out the optimal time point to replace several components simultaneously.

First extending the parameters in Section 2 below:

$\mathrm{C}_{1 \mathrm{i}}$ the cost caused by failure of component $\mathrm{i}$ of the system, $\mathrm{i}=1,2, \ldots \mathrm{n}$

$\mathrm{C}_{2 \mathrm{i}}$ the cost caused by replacement of component $\mathrm{i}$ of the system, $\mathrm{C}_{2 \mathrm{i}}<\mathrm{C}_{1 \mathrm{i}}, \mathrm{i}=1,2, \ldots \mathrm{n}$

$C^{(i)}(\mathrm{T})$ the cost rate per unit of time if it is replaced at time $\mathrm{T}$ of component $\mathrm{i}$, corresponding to the Eq.2, $\mathrm{i}=1,2, \ldots \mathrm{n}$

$\widehat{T}$ the optimal replacement time point of the whole system

Undergroup maintenance policy, every component costs a little more than their own optimal time point, but system down time frequency declines, which will make a difference if there is a start-up cost.

In the following parts, a simple method of calculating optimal time $\hat{T}$ is introduced. From Eq. 2 in Section 2, the cost rate can be got if time point for maintenance is given. Then the cost rate per unit of time of a system is given as Eq.4:

$\mathrm{C}(T)=\sum_{i=1}^{n} C^{(i)}(\mathrm{T})$ 
Where:

$$
C^{(i)}(T)=\frac{C_{1 i} * F_{i}(T)+C_{2 i} * \bar{F}_{i}(T)}{\int_{0}^{T} \overline{F_{l}(T)}} .
$$

Differentiating Eq.4 with respect to T and putting it equal to zero, then the optimal time point for maintaining of the system can be got. With the development of computer, enable us to get an approximate value of $\widehat{T}$ (An accurate value of $\widehat{T}$ is not needed, since that failure itself is a random event, it is impossible to know the accurate time of a failure, so we do not need to do the maintenance accurately. Besides, it is impractical in engineering field to exert the maintenance at a millisecond level, so an integer time can meet the requirement). T-C(T) graph can be got easily by MATLAB.By searching coordinate of the graph, an approximate value of $\widehat{T}$ can be got easily. Similarly, the approximate solution of Eq.2can be gotby the same method, while complicated derivation is not needed.

The Condition of ExertingGroup Maintenance Policy.Group maintenance policy is not always the optimal solution of the system. Only when there is a start-up cost and the start-up cost is very expensive, the group maintenance policy is the optimal choice. In following chapters, the condition of exerting group maintenance policy is researched.

Let $\mathrm{C}_{3}$ denotes start-up cost of the system. $\mathrm{EN}(\mathrm{t})$ denotes shutting down times of the system under age replacement policy, when the life cycle of the system is $\mathrm{t}$. EN'(t) denotes shutting down times of the system under group maintenance policy, when the life cycle of the system is t. $T_{i}^{*}$ denotes the optimal time for maintenance of component $i$ respectively $(i=1,2, \ldots n) . T_{\text {tot }}$ denotes life cycle time.

Then the total cost of a system if the maintenance is done respectively for every single component is showed in Eq.6:

$\operatorname{Cost}\left(T_{\text {tot }}\right)=\left(\sum_{i=1}^{n} C^{(i)}\left(T_{i}^{*}\right)\right) * T_{\text {tot }}+C_{3} * E N\left(T_{\text {tot }}\right)$

While total cost of group maintenance policy is:

$\operatorname{Cost}^{\prime}\left(T_{\text {tot }}\right)=\left(\sum_{i=1}^{n} C^{(i)}(\widehat{T})\right) * T_{\text {tot }}+C_{3} * E N^{\prime}\left(T_{\text {tot }}\right)$

According to Eq.6 and Eq.7, the condition of group maintenance policy is optimal choice is showed inEq.8:

$$
\operatorname{Cost}\left(T_{\text {tot }}\right)>\operatorname{Cost}^{\prime}\left(\left(T_{\text {tot }}\right)\right. \text {. }
$$

SubstituteEq.6 and Eq.7 into Eq.8, the condition ofgroup maintenance policy is the optimal choice is displayed in Eq.9: 
$C_{3}>\frac{\left(\sum_{i=1}^{n} C^{(i)}(\widehat{T})\right) * T_{\text {tot }}-\left(\sum_{i=1}^{n} C^{(i)}\left(T_{I}^{*}\right)\right) * T_{\text {tot }}}{E N\left(T_{\text {tot }}\right)-E N^{\prime}\left(T_{\text {tot }}\right)}$.

The value of unknown parameter in Eq.9can be got by simulation software, so complicated derivation is not needed. In Section 4, a numerical example is presented, thencombining with simulation software, the optimal results can be got.

\section{Numerical example}

System simulation can simulate different situations during the life cycle of the system, and it is an important tool in engineering field. A software named VISUALSPARis used in this paper, which is a powerful software for simulation. It can simulate the system during the life cycle, and can define logic by users themselves, so it isa convenientand practical software for system simulation.

Verification of the Single Component Age Replacement Policy.In order to increase the credibility of the algorithm in Section 2, it is necessary to validate the single component age replacement policy. Suppose that a component subjects to Weibull distribution with shape parameter $\mathrm{m}=4$, characteristic $\eta=300$. It costs $\$ 500$ due to failure while costs $\$ 30$ due to replacement, i.e. $C_{1}=500$, $\mathrm{C}_{2}=30$. Substitutethese parameter into Eq.3, an optimal time $\mathrm{T}^{*}=114.64$ for maintaining can be got. Substitute $T^{*}$ into Eq.2, we can get cost rate per unit of time is $\$ 0.3497$, i.e. if we exert replacement at $\mathrm{T}^{*}=114.64$ for the component, we need to pay $\$ 0.3497$ per unit of time during the life cycle of the component. All of the parameters and results of calculating are displayed in Table 1.

By simulating the process, input the data above, and set the life cycle is $10000 \mathrm{~h}$. Then set the replacement time at $80 \mathrm{~h}, 100 \mathrm{~h}, 114 \mathrm{~h}, 150 \mathrm{~h}$ and $200 \mathrm{~h}$ respectively and get the outputs in Fig.1.

An intuitive result can be got by Fig.1: if we exert the replacement at 114h, we can get the minimal cost, which is $\$ 3465.42$, very close to the result we calculated in Eq.2, so the age replacement policy for a single component is correct.

Table 1 Parameters of a single component

\begin{tabular}{cc}
\hline Parameter & Value \\
\hline $\mathrm{m}$ & 4 \\
$\eta$ & 300 \\
$\mathrm{C}_{1}$ & 500 \\
$\mathrm{C}_{2}$ & 30 \\
$\mathrm{~T}^{*}$ & 114.64 \\
$\left.\mathrm{C}^{*}\right)$ & 0.3479 \\
\hline
\end{tabular}




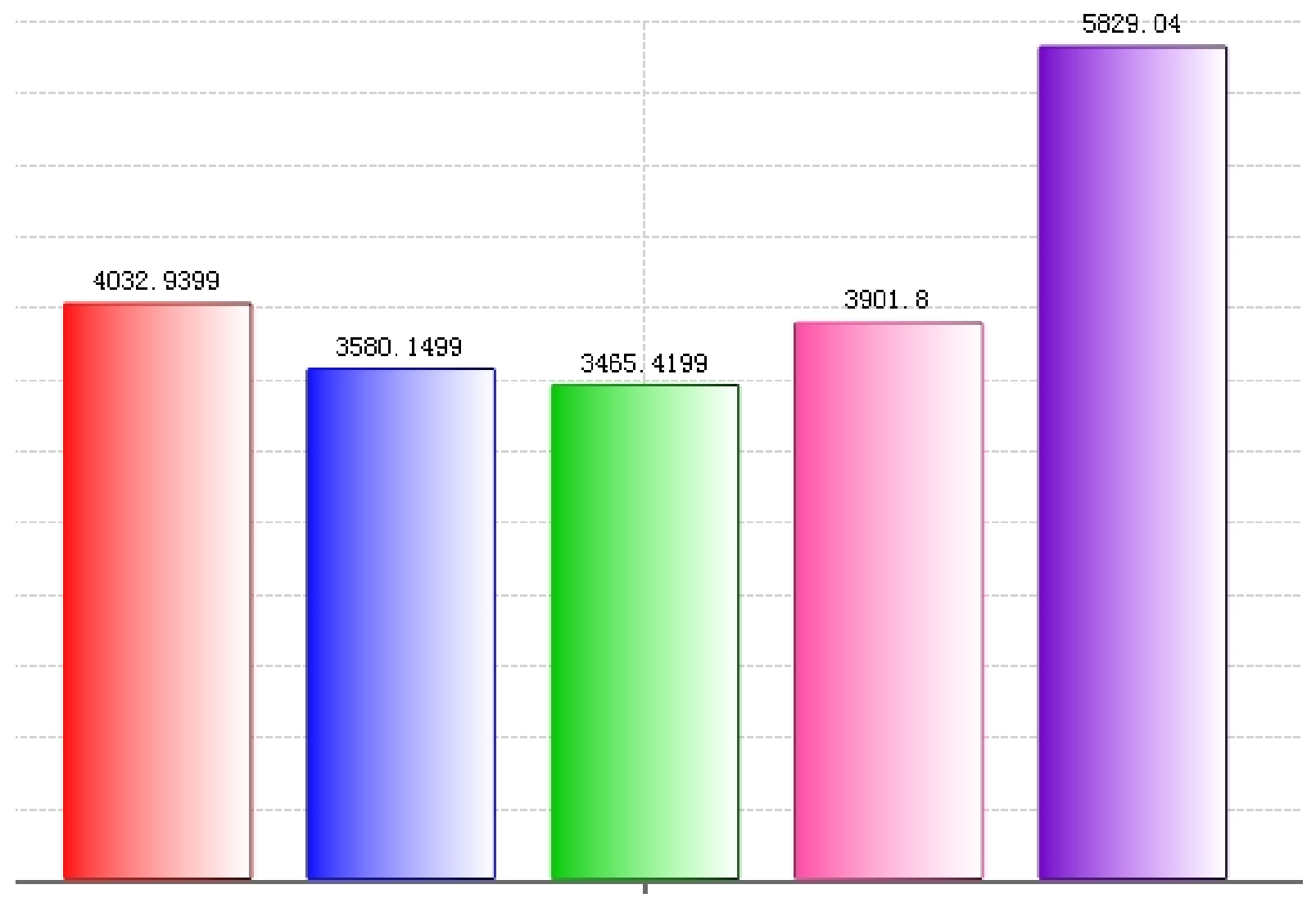

Fig. 1 Costs under different replacement policies

Maintenance for Multi-Components System.Suppose thatthree components need to maintain simultaneously, and these components all subject to Weibull distribution, their parameters are listed inTable 2.

Substitute these parameters in Table 2 into Eq.4, an expression with respect to $\mathrm{T}$ can be got, which means the cost rate per unit of time by different $\mathrm{T}$. Defining an array contains 1 to 100, step length is 1 , then a corresponding array for $\mathrm{C}(\mathrm{T})$ can be got.Afunction diagram with $\mathrm{T}-\mathrm{C}(\mathrm{T})$ can be drawn by MATLAB, which is showed in Fig. 2 .

Table 2 Parameters of the components of the system

\begin{tabular}{cccc}
\hline $\mathrm{n}$ & 1 & 2 & 3 \\
\hline $\mathrm{m}$ & 4 & 5 & 4 \\
$\eta$ & 300 & 100 & 150 \\
$\mathrm{C}_{1 \mathrm{i}}$ & 500 & 500 & 600 \\
$\mathrm{C}_{2 \mathrm{i}}$ & 30 & 50 & 60 \\
$\mathrm{~T}_{\mathrm{i}}^{*}$ & 114.64 & 48.86 & 65.87 \\
$\mathrm{C}^{*} \mathrm{~T}_{\mathrm{i}}{ }^{*}$ & 0.3497 & 1.2822 & 1.2191 \\
\hline
\end{tabular}




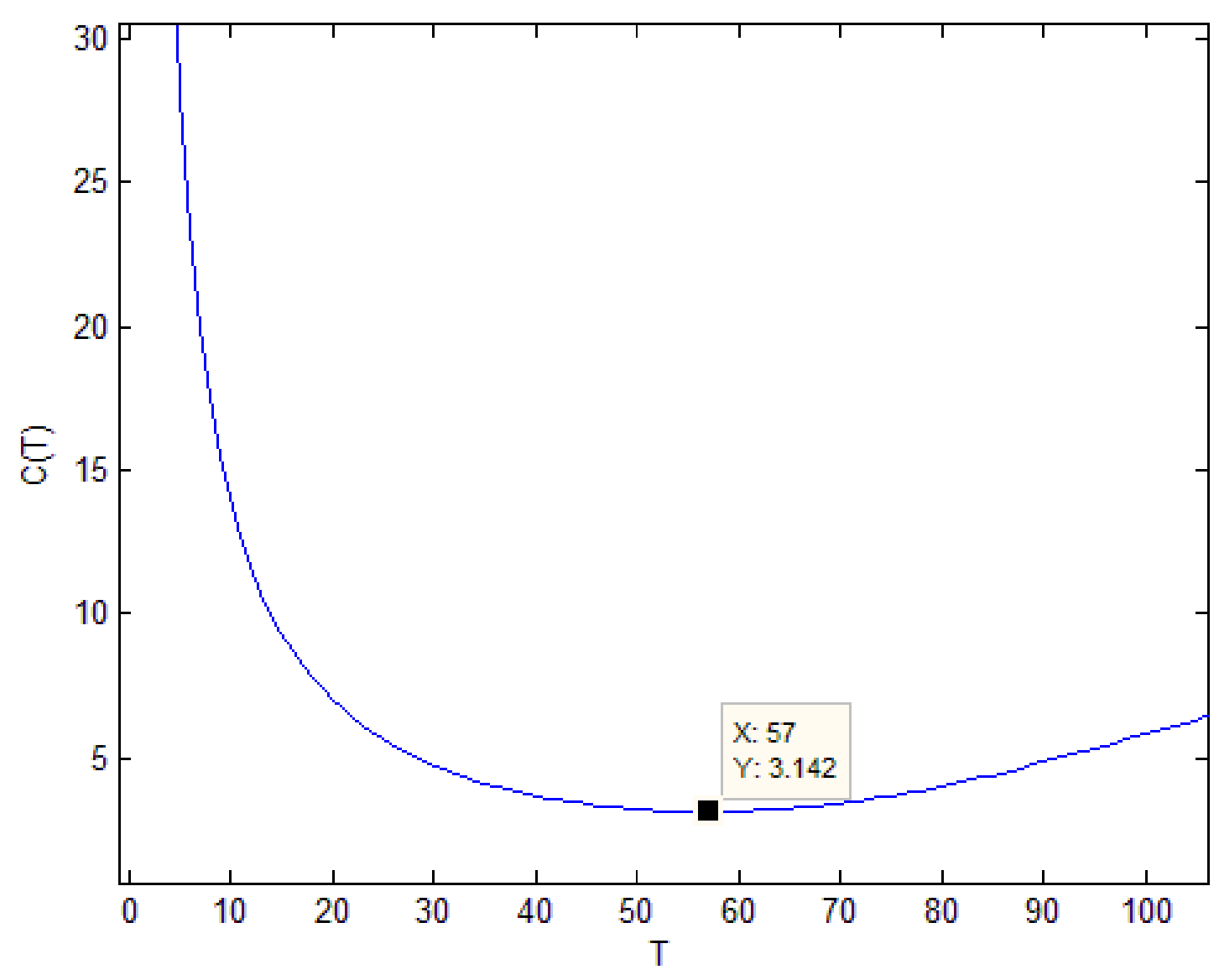

Fig.2 Function diagram for T-C(T)

From Fig. 2 we can see the optimal time of the system is $57 \mathrm{~h}$, and the cost rate per unit of time is $\$ 3.142$.

The Influence of Start-up Cost.As have mentionedin Section 3.2, group maintenance policy is not always the optimal solution for maintenance. Only when there is a start-up cost and it is expensive enough that group maintenance policy is an optimal solution. This part using the example in Section 4.2 and show when group maintenance policy is cheaper than single component age replacement policy.

Using simulation software the parameters we need in Eq.9 can be easily got. After simulation, the results are displayed in Table 3.

Table 3Results of the simulation

\begin{tabular}{ccc}
\hline & Respectively & Group \\
\hline Simulation Time[h] & 10000 & 10000 \\
Cost $[\$]$ & 28361 & 31456 \\
System down times & 444.7 & 177.4 \\
\hline
\end{tabular}


Substituting the data in Table 3 into Eq.9, the result is displayed below:

$C_{3}>\frac{\left(\sum_{i=1}^{n} C^{(i)}(\widehat{T})\right) * T_{\text {tot }}-\left(\sum_{i=1}^{n} C^{(i)}\left(T_{I}^{*}\right)\right) * T_{\text {tot }}}{E N\left(T_{\text {tot }}\right)-E N^{\prime}\left(T_{\text {tot }}\right)}=10.8$

To sum up, if the start-up cost of the system is more than $\$ 10.8$, then group maintenance policy is the optimal choice, while we should choose single age replacement policy if the start-up cost is less than $\$ 10.8$.

\section{Conclusion}

On the basis of age replacement policy, this paper studies group maintenance policy further, which can apply to the system with start-up cost.In this paper, the following result is given: under what condition the group maintenance policy is optimal when taking start-up policy into account. Besides, the assumption in this paper is the replacement is instantaneous, so we have not taken down time into account. If the down time of replacement can't be ignored, then group maintenance policy may have a greater chance to be the optimal maintenance policy. The other advantage of group maintenance is its higher availability(Since it has lower down times frequency). Another assumption of this paper is that we have enough workers to exert the maintenance, however, if this assumption is not true, the number of workers is limited, so we do not want to exert maintenance too often, then thegroup maintenance policy should be considered.

If there are too many components in a system, the single time point for group maintenance may be not enough, since the optimal time of each component is very different. Under this condition, a model with multi time point for group maintenance may be researched in the future.

\section{Reference}

[1] Cox D R. Renewal Theory[J]. Encyclopedia of Statistical Sciences, 1962, 4(01):281-302.

[2] Glasser G J. The age replacement problem[J]. Technometrics, 1967, (1):83.

[3] Fox B. Age replacement with discounting[J]. Operations Research, 1966, 14(3).

[4] Block H W, Borges W S, Savits T H. A general age replacement model with minimal repair[J]. Naval Research Logistics, 1988, 35(5):365-372.

[5] Oku Sheu S H, Kuo C M, Nakagawa T. Extended optimal age replacement policy with minimal repair[J]. RairoRechercheOpérationnelle, 1993, 27:337-351. moto K, Elsayed EA (1983) An optimal group maintenance policy. Nav Res Logist Q 30:667-674.

[6] JingjieXun. Hydropower Generators Start-up Costs and Control Analysis in Power Market[D]. Sichuan University, 2005. DOI:doi:10.7666/d.y779560.

[7] Horenbeek A V, Pintelon L. A dynamic predictive maintenance policy for complex multi-component systems[J]. Reliability Engineering \& System Safety, 2013, 120(12):39-50. 
[8] Barlow R E, Proschan, Frank, Hunter, Larry C. Mathematical theory of reliability[M]// SIAM, 1996.

[9] Toshio Nakagawa. Maintenance theory of reliability[J]. Springer, 2005. 